\title{
GMR
}

\section{Verification and evaluation of grain QTLs using RILs from TD70 x Kasalath in rice}

\author{
Y.D. Zhang ${ }^{1,2}$, J. Zheng ${ }^{2}$, Z.K. Liang ${ }^{3}$, Y.L. Liang ${ }^{1}$, Z.H. Peng ${ }^{3}$ and C.L. Wang ${ }^{1,2}$ \\ ${ }^{1}$ College of Agriculture, Nanjing Agricultural University, Nanjing, China \\ ${ }^{2}$ Institute of Food Crops, Jiangsu Academy of Agricultural Sciences, Nanjing, China \\ ${ }^{3}$ Department of Biochemistry, Molecular Biology, Plant Pathology and Entomology, \\ Mississippi State University, Starkville, MS, USA \\ Corresponding author: C.L. Wang \\ E-mail: cailin_wang@sina.com \\ Genet. Mol. Res. 14 (4): 14882-14892 (2015) \\ Received April 9, 2015 \\ Accepted July 24, 2015 \\ Published November 18, 2015 \\ DOI http://dx.doi.org/10.4238/2015.November.18.53
}

ABSTRACT. Grain size is an important trait that directly influences the yield of rice. Validation and evaluation of grain genes is important in rice genetic studies and for breeding. In a population of 240 recombinant inbred lines (RILs) derived from a cross between an extra-large grain japonica variety TD70 and a small grain indica variety Kasalath, we mapped 19 QTLs controlling grain traits. These QTLs included six cloned grain genes, namely, GW2, GS3, qSW5, qGL3, GS5, and GW8. All of the alleles with the optimal effects on grain size came from TD70, the variety with extra-large grains. To verify these gene loci, we cloned and sequenced GW2, GS3, GW5 (qSW5), qGL3, GS5, GW8, and TGW6 in TD70 and Kasalath, and found several functional polymorphisms in the sequences of the genes. New functional markers for the cloned genes were designed to identify parents and RILs. The contributions of these polymorphisms to the improvement in rice grain size traits were evaluated. Our results indicate that at least six functional polymorphisms have additive effects on grain shape and that one non-functional polymorphism in TGW6 affects grain shape in TD70. The newly designed markers will be useful in further studies to identify 
functional grain genes. Our findings provide insight into the control of grain size in rice, and they will be of value for improving rice grain yield.

Key words: Rice; Grain gene; Molecular markers; Verification; Effect evaluation

\section{INTRODUCTION}

Quantitative trait loci (QTLs) are essential to the development of agronomically important factors such as grain length, width, and thickness, which have significant impacts on the yield and quality of rice crops (Xing and Zhang, 2010; Ying et al., 2012). Multiple QTLs and genes controlling rice grain size have been mapped on the 12 chromosomes using molecular markers and different mapping populations (Huang et al., 2013). Several genes controlling grain size have been analyzed (Zuo and Li, 2014), for example, GS5 (Li et al., 2011), GS3 (Fan et al., 2006; Mao et al., 2010), GW2 (Song et al., 2007), GW5 (Weng et al., 2008), qSW5 (Shomura et al., 2008), qGL3 (Hu et al., 2012; Qi et al., 2012; Zhang et al., 2012), GW8 (Wang et al., 2012) and TGW6 (Ishimaru et al., 2013), and qGL7 and GS7 (Bai et al., 2010; Shao et al., 2012). Although a number of genes for grain size have been cloned, the underlying molecular mechanisms and the potential interaction of these genes in grain size regulation remain to be elucidated. A major problem is that these genes or QTLs were analyzed on different genetic backgrounds. A precise assessment of the function of each gene and the potential interaction of the genes in grain size regulation requires that the QTLs and genes be on the same genetic background. To this end, it is necessary to identify rice germplasms that contain multiple QTLs with additive effects on grain phenotype. Previously, we used data obtained in the 2010 and 2011 growing seasons to map 18 QTLs that control grain traits, and to clone five genes with effects on grain phenotype, namely, GW2, GS3, qSW5, qGL3, and GS5. All of the alleles for increased grain size were derived from TD70, the variety with extralarge grains (Zhang et al., 2013). QTLs for GW8 and TGW6 were not detected (Wang et al., 2012; Ishimaru et al., 2013); however, the former was detected using data obtained in the 2012 and 2013 growing seasons (Fiqure S1). The aims of the present study were to sequence the seven genes for grain size that had previously been cloned, to develop further molecular markers for analysis of grain traits, and to elucidate the role of the identified genes in grain size characteristics. Our findings will be of value to the understanding of grain size regulation and for breeding programs to improve grain size in rice based on molecular markers.

\section{MATERIAL AND METHODS}

\section{Plant materials and growth conditions}

A recombinant inbred line (RIL) population consisting of 240 lines was developed from a cross between the japonica variety TD70, an extremely large grain variety derived from Tiane-gu///9520//(72-496/Yu-nuo), and the indica variety Kasalath, a small grain sized variety (Zhang et al., 2013). The parental varieties TD70 and Kasalath along with the 240 RIL lines were grown in two replicate plots in the experimental field of the Institute of Food Crops, Jiangsu Academy of Agricultural Sciences, China, in 2012 and 2013. Seeds were harvested immediately upon maturation and wind dried for 30 days to maintain a water content of approximately $14 \%$. 


\section{Phenotype characterization}

The length, width, and thickness of the rice grains were measured. Ten seeds from each plant were selected at random and the three traits were measured using a Vernier caliper (accurate to $0.01 \mathrm{~mm}$ ). The mean of each trait for five plants of each genotype was used as the phenotypic value.

\section{Primer design}

The genomic sequences of GW2, GS3, GW8, qSW5 (GW5), GS5, qGL3, and TGW6 were downloaded from the NCBI website (http://www.ncbi.nlm.nih.gov/). Primers were designed to amplify alleles from both TD70 and Kasalath genetic backgrounds (Table S1).

\section{PCR amplification, electrophoresis, and product sequencing}

The primers were used to amplify alleles from both parental varieties and amplicon sizes were verified using $2 \%$ agarose gel electrophoresis. Targeted DNA bands were cut from the gel and recovered using an AxyPrep DNA Extraction Kit. The purified PCR products were then sequenced. High quality forward and reverse reads were assembled and the consensus sequence obtained was used to compare the parental varieties using the DNAman software.

\section{Molecular markers and amplified products}

Three dCAP (derived cleaved amplified polymorphic sequence) markers for GW2, qGL3, and GW8, two CAP (cleaved amplified polymorphic sequence) markers for GS3 and TGW6, an SSR (simple sequence repeat) marker for GS5, and an indel (insertion/deletion) marker for qSW5 (GW5) were used to identify sequence differences between TD70 and Kasalath. The primer sequences for each of these markers are listed in Table S2. PCR amplification conditions were: 4 min at $94^{\circ} \mathrm{C}$ : 33 cycles of $94^{\circ} \mathrm{C}$ for $30 \mathrm{~s} ; 49^{\circ} \mathrm{C}$ (GS3, qGL3, and GW8), $58^{\circ} \mathrm{C}(T G W 6), 58.5^{\circ} \mathrm{C}$ (qSW5), $52^{\circ} \mathrm{C}$ (GW2), and $68^{\circ} \mathrm{C}$ (GS5) for $30 \mathrm{~s} ; 72^{\circ} \mathrm{C}$ for $1 \mathrm{~min}$; and a final extension at $72^{\circ} \mathrm{C}$ for $10 \mathrm{~min}$. The restriction endonucleases and the reaction conditions used here are detailed in Table S3. Amplification products were separated using either a $4 \%$ denatured polyacrylamide gel or a $3 \%$ agarose gel and visualized by silver staining or ethidium bromide, respectively.

\section{RESULTS}

\section{Grain size trait identification in RIL population and parental varieties}

As described above, we used the japonica variety TD70 and the indica variety Kasalath to construct an RIL population. In 2012, the mean value in TD70 for grain length was $13.40 \mathrm{~mm}$, mean grain width was $4.42 \mathrm{~mm}$, and mean grain thickness was $2.99 \mathrm{~mm}$; the 1000 -grain weight was $65.0 \mathrm{~g}$. In Kasalath, these four phenotypic values were $8.04 \mathrm{~mm}, 2.48 \mathrm{~mm}, 1.91 \mathrm{~mm}$, and 17.4 $\mathrm{g}$, respectively. The parental varieties showed no significant differences for these phenotypic values between the 2012 and 2013 growing seasons (Figure 1). The RIL population had an average grain length of $9.83 \mathrm{~mm}$, width of $3.12 \mathrm{~mm}$, thickness of $2.11 \mathrm{~mm}$, and 1000 -grain weight of 31.2 
g. Grain length in RILs showed a continuous multi-peaked distribution pattern suggesting control by major genes (Figure 2A); in contrast, grain width, grain thickness, and 1000-grain weight showed continuous single-peaked distribution patterns (Figure 2B, C, and D). The three seed traits showed similar frequency distributions in 2012 and 2013. The data confirm the extremely large grain size in TD70 and small grain size in Kasalath; the RIL population had an intermediate size range and thus showed transgressive segregation from the smaller size in Kasalath.
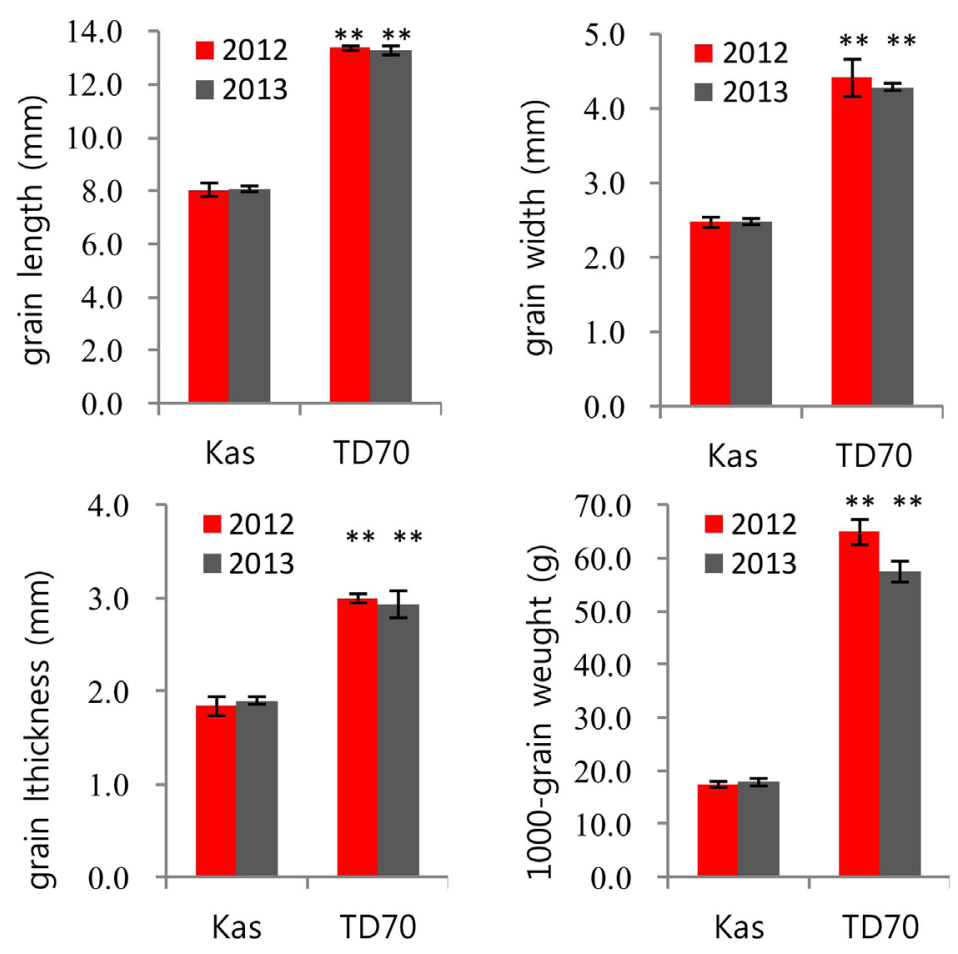

Figure 1. Comparison of the grain phenotypes of the parental strains TD70 and Kasalath (Kas). ${ }^{*}$ Significant difference at the 0.01 level in the $t$-tests.

\section{Gene sequence analysis}

According to the mapping results in the 2012 and 2013 growing seasons (Figure S1), 19 QTLs controlling grain traits were detected on chromosomes 2, 3, 5, 7, 8, and 9; these QTLs included six cloned genes with effects on grain characteristics, namely GW2, GS3, qSW5, qGL3, GS5, GW8, and also $q G L 7$ and GS7. The grain length genes GS3 and $q G L 3$ are located within the marker interval RM6080 - RM6832 on chromosome 3 (Zhang et al., 2013). We amplified GS3 from TD70 and Kasalath using the specific primers listed in Table S1. Several sequence variations are present in the GS3 alleles of the two varieties (Figure S2). A C-to-A transversion at the +165 position in the 2nd exon is a critical mutation that alters the function of the gene (Fan et al., 2006). The nucleotide at this position is A in TD70 but C in Kasalath (Figure 3A), indicating that TD70 carries a loss-of-function mutation at this locus, whereas Kasalath has the wild-type allele. 
We also sequenced the $q G L 3$ gene in the two varieties. The $q G L 3$ allele in TD70 was 8700 bp and in Kasalath was 8695 bp; the two alleles shared 99.56\% identity (Figure S2). A C-to-A transversion at the +1092 position in the 10th exon was shown to cause a change of function in qGL3 (Zhang et al., 2012). At this position in TD70, an A nucleotide was present, whereas in Kasalath, a $\mathrm{C}$ nucleotide was found; thus, TD70 carries a loss-of-function allele, whereas Kasalath has the wild-type allele (Figure 3B).
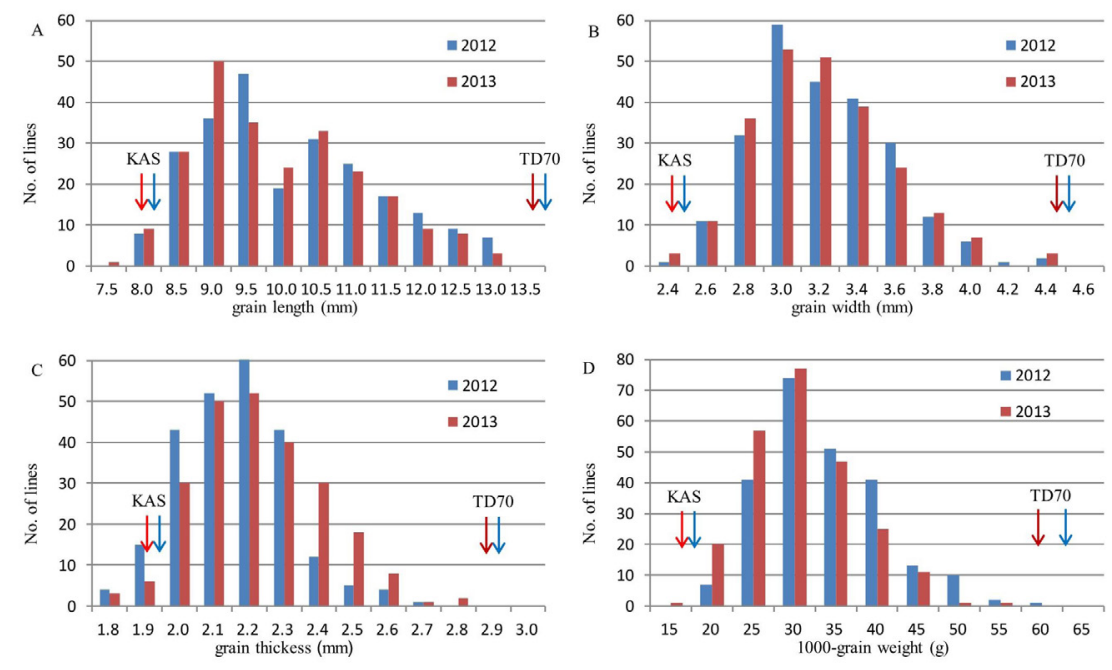

Figure 2. Variation for the four grain traits measured here in the RIL population in the 2012 and 2013 growing seasons.

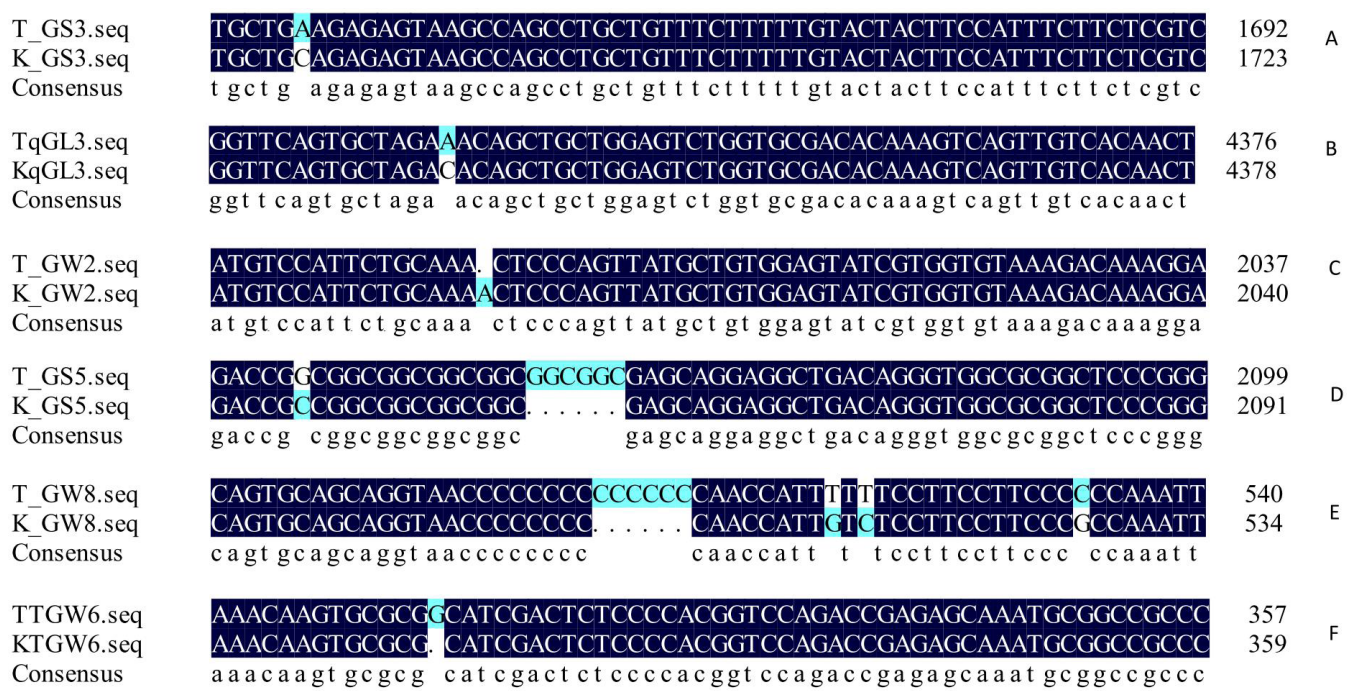

Figure 3. Comparison of nucleotide sequences in GS3, qGL3, GW2, GS5, GW8, and TGW6 genes from TD70 and Kasalath (Kas). A.-F. Partial alignments of the six genes sequences highlighting differences between the two parental alleles. 
Sequencing of GW2 indicated that the TD70 allele was $6629 \mathrm{bp}$, while that of Kasalath was 6632 bp. The alleles shared $99.67 \%$ similarity with differences in a few single nucleotides or indels. The coding sequence for GW2 in the rice variety WY3, used in the initial gene cloning, had a deletion of an A at the +316 position; this deletion causes a reading frame shift that resulted in only 115 amino acids being translated from the sequence. We found that GW2 of TD70 had the same mutation, whereas the Kasalath allele expressed the full-length 425 amino acid protein due to lack of a mutation (Figure 3C).

Polymorphisms and allelic variations in the promoter regions of GS5 and GW8 have been suggested to induce increased expression of the genes, which then results in production of wider and heavier grains (Li et al., 2011; Wang et al., 2012). Comparison of the GS5 sequences in TD70 and Kasalath identified a deletion in the latter consisting of six bases (GCGGCG) at the +2057 position that encode two alanine residues (Figure 3D). This six-base deletion mutation was also shown to distinguish the Zhenxian 97 variety (wild-type sequence, wide grain size) and H94 variety (6-base deletion, narrow grain size). The GW8 sequences of TD70 and Kasalath identified base differences or indels (Figure 3E) that underlie the Kasalath narrow size grain. The TGW6 gene of Kasalath has a positive regulatory role (Ishimaru et al., 2013); comparison of the gene sequence with that of TD70 identified a single base pair deletion at nucleotide 313, which nevertheless resulted in a functional TGW6 allele in Kasalath (Figure 3F).

Three qSW5 alleles have been identified, namely, Kasalath-type qSW5, indica-II type qSW5, and Nipponbare-type qSW5, which identified a 1971 bp product, 1021 bp product, 759 bp product by the molecular marker N1212del, respectively (Shomura et al., 2008; Yan et al., 2011). The gene qSW5 was cloned in a cross between Kasalath and Nipponbare varieties. Here, we tested the amplification band using this marker and found that it identified a 759-bp product in TD70 (Figure 4). This result showed allelic types of TD70 was identified to be Nipponbare-type qSW5, not to be indica-II type qSW5 at the qSW5 (Kasalath-type) locus.

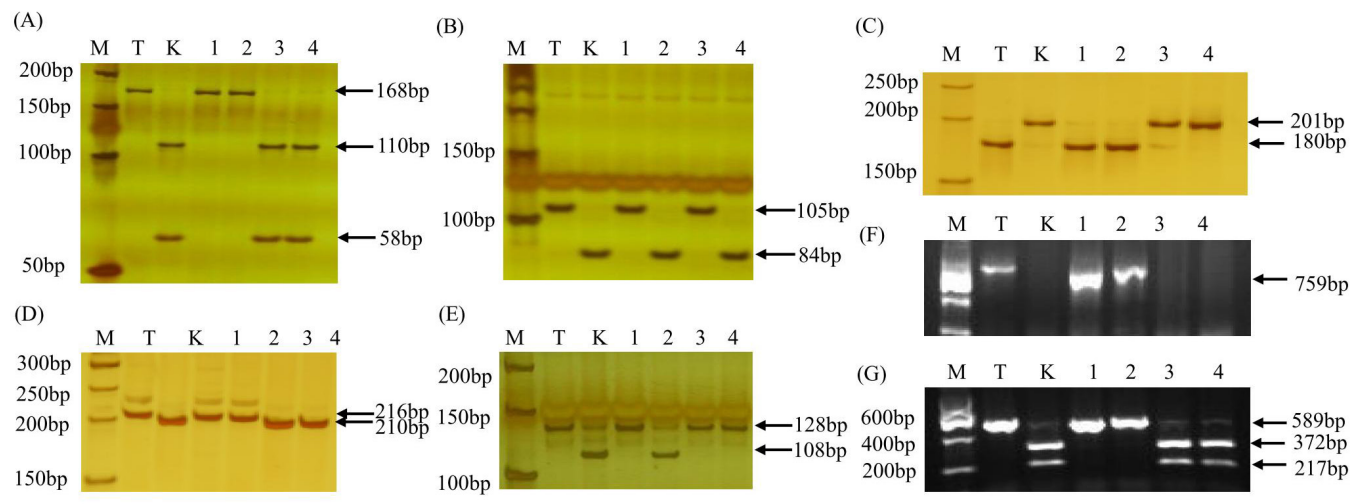

Figure 4. PCR amplification and enzyme reaction products of GS3 (A), qGL3 (B), GW2 (C), GS5 (D), GW8 (E), qSW5 (F), and TGW6 (G) in TD70, Kasalath and four RILs. Line $M=$ DNA size marker; line $T=$ TD70; line $K=$ Kasalath; lines 1-4 = RILs. Small fragments are not shown in B (21 bp), C (21 bp), and E (20 bp) as they were too far from the main fragment.

\section{Designing markers for functionally polymorphic genes}

Based on identification of sequence polymorphisms in the GW2, GS3, qGL3, GS5, GW8, and TGW6 genes that affect their comparative function in TD70 and Kasalath varieties, we created 
four types of marker to distinguish these polymorphic variants. We designed the specific primers and identified favorable restriction endonucleases. After PCR, the GS3 CAPS marker product was specifically cleaved by Pstl: TD70 gave a 168 bp fragment and Kasalath gave 110 bp and 58 bp fragments (Figure 4A). Accl digestion of the qGL3 dCAPS marker gave 21 bp and 84 bp fragments in Kasalath; however, TD70 yielded a single amplification product of 105 bp (Figure 4B). The GW2 dCAPS marker sequence in Kasalath produced only a $201 \mathrm{bp}$ fragment, while in TD70 it could be specifically cleaved into $180 \mathrm{bp}$ and $21 \mathrm{bp}$ fragments (Figure 4C). An SSR marker for GS5 amplified a 216-bp fragment in TD70 and a 210-bp fragment in Kasalath (Figure 4D). After endonuclease digestion, the GW8 dCAP yielded 20- and 108-bp fragments in Kasalath (Figure 4E).

The qSW5 indel was approximately 759 bp in TD70, but no fragment was recovered from Kasalath (Figure 4F). The TGW6 CAP marker yielded 217- and 372-bp fragments in Kasalath but not TD70 (Figure 4G). These various markers can therefore accurately identify different grain genotypes in RILs.

\section{Grain size differences generated by alleles with different functionality}

The molecular markers described above were used to screen functional polymorphisms in the seven genes in RILs. We found that 77 RILs carried GW2TD70, 109 carried GS3 ${ }^{T D 70}, 82$ carried $q G L 3^{T D 70}, 122$ carried $G S 5^{T D 70}, 143$ carried $G W 8^{T D 70}, 86$ carried $G W 5^{T D 70}$, and 140 carried TGW6 ${ }^{T D 70}$ (Table S4). The disparity in the number of RILs with different genotypes may simply be due to the limited population size. We next evaluated the effects on grain traits produced by the different haplotypes derived from TD70 and Kasalath.

Our analysis indicated that $q G L 3$ and GS3 were associated with grain length, while GW2, qSW5 (GW5), GS5, and GW8 were associated with grain width. Data produced in the 2013 growing season indicated that the TD70 alleles for $q G L 3$ and $G S 3$ added $1.83 \mathrm{~mm}$ and $1.69 \mathrm{~mm}$ to grain length compared to the Kasalath alleles (Figure 5A). Similarly, the TD 70 alleles for GW2, GS5, GW5, and TGW6 increased average grain widths by $0.51,0.30,0.31$, and $0.01 \mathrm{~mm}$, respectively (Figure 5B). Except for GW8, the genes showed significant differences on phenotypic values of grain thickness (Figure 5C). For 1000-grain weight, TD70 alleles induced a significantly higher mean, with the exception of GW8 and TGW6 (Figure 5D). Lines carrying the TD70 alleles $q G L 3^{T D 70}, G S 3^{T D 70}$, and $G W 2^{T D 70}$ showed $7.09,6.39$, and $5.54 \mathrm{~g}$ increases in 1000-grain weight, respectively.

In summary, the TD70 alleles for $q G L 3, G S 3, G W 2$, and GS5, followed by qSW5 (GW5) had the greatest phenotypic effects compared to their Kasalath counterparts. Conversely, GW8 and TGW6 made the least contribution to the differences between the two varieties (Figure 5). A similar conclusion could also be made from the data obtained during the 2012 growing season (Table S4).

\section{DISCUSSION}

\section{QTL validation}

In essence, QTLs are a genetic feature defined from the perspective of statistics. The QTL map is based on probabilities and only indicates which regions might be associated with genes that influence quantitative traits. However, for a given QTL, the number of genes involved is unknown and their role in determining quantitative traits is uncertain (Borevitz and Chory, 2004). Currently, the published data on the Gramene website (http://www.gramene.org/) for 2013 lists at least 300 

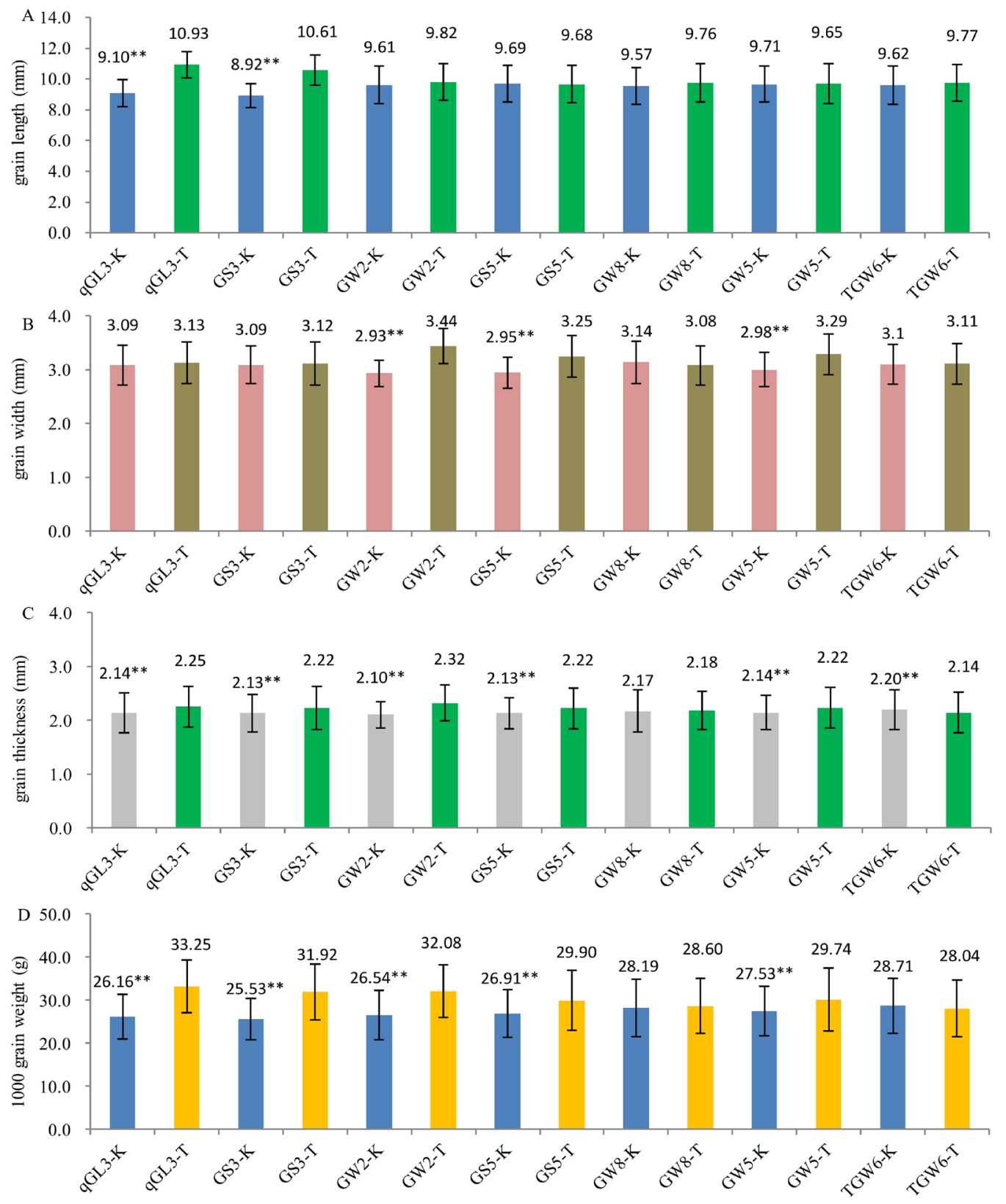

Figure 5. Comparison of the genetic effects of GS3, qGL3, GW2, GS5, GW8, GW5, and TGW6 from TD70 and Kasalath on grain length (A), grain width (B), grain thickness (C), and 1000-grain weight (D). The data are reported as means \pm SD. Line $T=$ TD70; line $K=$ Kasalath. ${ }^{*}$ Significant difference at the 0.01 level in the $t$-tests.

QTLs related to grain size or weights that are mapped throughout the 12 chromosomes of rice. However, very few genes have actually been cloned or fine mapped. In addition to the six cloned 
grain size genes, GW2, GS3/qGL3, GS5, GW5 (qSW5), and GW8, and two fine mapped grain size loci, only six more QTLs were identified in the single growing season. Further analyses need to be performed to determine whether these loci are associated with genes for grain size. In this study, we mapped QTLs and validated major genes in corresponding regions of QTLs.

\section{Functional polymorphisms in TD70}

Lu et al. (2013) sequenced GW2, GS5, and qSW5 alleles in the germplasm of 127 varieties of rice (Oryza sativa) and 10-15 samples of wild rice (Oryza rufipogon) and identified 10 GW2 haplotypes, 10 GS5 haplotypes, and 15 qSW5 haplotypes. Grain size genes can exhibit sequence variation in different germplasms and show different haplotypes, but a key functional mutation is often present in a specific site or region. GW2 of TD70 encodes a protein with the same number of amino acids as that encoded in the WY3 variety (Song et al., 2007). The GW2 gene in these two varieties also performs the same function. The Kasalath GW2 allele shows $99.92 \%$ similarity to the sequence deposited in the database (GenBank accession No. EF447275). A T-to- $C$ transition occurred at the +114 position with no change in amino acid composition. Another key C-to-A transversion at the +165 position of the coding region of the 2 nd exon causes an alteration in GS3 ${ }^{M H 63}$ function (Fan et al., 2006; Mao et al., 2010). TD70 contains this mutation, but not Kasalath. Comparison of the $q G L 3$ sequences of TD70 and Kasalath identified a C-to-A transversion at the +1092 position of the 10th exon of the former that was associated with differences in function (Zhang et al., 2012). Therefore, TD70 contains mutations that influence the function of all three genes, whereas Kasalath has the normal wild-type alleles. Many differences were observed between the GS5 and GW8 sequences of TD70 and Kasalath. According to our phenotypic analysis, the GS5 and GW8 alleles of TD70 had additive effects. GW5 (qSW5) of TD70 was identical to qSW5 of Nipponbare, while the TD70 TGW6 had the same sequence as the Kasalath allele. Two fine mapped loci, GL7 and GS7, require more information for their identification. We showed that three dCAP markers for GW2, qGL3, and GW8, two CAP markers for GS3 and TGW6, and an SSR marker for GS5 genes could successfully identify the parents and distinguish RILs based on functional polymorphisms.

\section{qGL3, GW2, and GS3 play important roles in regulation of rice grain size}

Considerable progress has been made in the categorizing of GS3, GW5, GW2, GS3, GS5, and TGW6 haplotypes (Mao et al., 2010; Takano-Kai et al., 2009; 2011; Dixit et al., 2013; Ishimaru et al., 2013; Lu et al., 2013), but only one haplotype having positive functional polymorphic sites had been tested to contributes more significantly effect on grain traits than other haplotypes (Song et al., 2007; Mao et al., 2010; Zhang et al., 2012). In this study, we showed that the TD70 qGL3, GS3, GW2, GS5, and qSW5 (GW5) alleles had a positive effect on grain weight compared to their Kasalath counterparts. In particular, $q G L 3, G W 2$, and GS3 played important roles in regulating rice grain size in RILs. GW2 was previously reported to have a large effect on grain width (Song et al., 2007), our results showed that lines carrying $G W 2^{T D 70}$ had a large increase in grain width compared to the Kasalath allele. A similar result has been described for $q G L 3$ (Zhang et al., 2012), and alleles with positive effects on grain size, for example, GS3, qSW5 (GW5), GS5, and GW8, are common in modern rice varieties (Wang et al., 2012; Lu et al., 2013). An RIL with the rare combination of mutations in $q G L 3$ and $G W 2$ will be invaluable for improvement in rice grain size. 


\section{GW8 and TGW6 do not have significant effects}

Seed size is a complex quantitative trait in crops and any attempt to introduce genetic improvements by means of "gene pyramiding" are likely to be difficult to achieve. The intricate and complicated interactions of the genes that contribute to seed size are the principal obstacle for such gene pyramiding (Yan et al., 2011). The QTL GW8 positively regulates grain width and weight via the promotion of cell proliferation. GW8 encodes the transcription factor OSSPL16 that positively regulates the expression of several genes involved in the G1-to-S transition. A higher expression level in GW8 promotes cell division and grain fithat, thereby increasing grain width and yield. GW8 generated a $13.9 \%$ advantage for NIL-GW8 with respect to 1,000-grain weight (Ishimaru et al., 2013). Some studies have indicated that GS3 and GW8 act in independent pathways (Ishimaru et al., 2013). TD70 appears to have "pyramided" many genes of value to grain phenotype. The effect of the GW8 ${ }^{T D 70}$ might be blocked by the those of other additive genes; this may also prevent any advantage in terms of grain weight gain from GW8 of Kasalath..

A similar phenomenon was found for TGW6. The Kasalath tgw6 allele carries a premature stop codon caused by a frame-shift mutation; NIL tgw6 plants have a remarkably reduced level of free indole acetic acid. Taking into account the relatively low grain weight of Kasalath, it is not surprising that $T G W 6^{\text {Kasalath }}$ and $T G W 6^{T D 70}$ made no significant difference on phenotypic values because of the influence of interactions with other genes. A clearer understanding of the gene effects on controlling grain growth and development will accelerate the process of optimization of gene pyramiding for rice breeding.

In summary, we verified the presence of functional polymorphisms in the GW2, GS3, GW5 (qSW5), qGL3, GS5, GW8, and TGW6 alleles of TD70 and Kasalath. We have developed new functional markers for these cloned genes that enable discrimination of parental varieties and RILs. Additionally, we evaluated the different genetic effects for improving rice grain size traits depending on functional and non-functional alleles. Our results indicated that at least six functional genes with additive effects on grain phenotype and one wild non-functional gene, TGW6, that influences grain shape have been accumulated in TD70. This suggests that TD70 might be of value for a gene pyramiding approach to generation of larger rice grain sizes.

\section{Conflicts of interest}

The authors declare no conflict of interest.

\section{ACKNOWLEDGMENTS}

Research supported by the National Natural Science Foundation of China (Grant \#31271678), the Jiangsu Agricultural Scientific Self-Innovation Fund [Grant \#CX(12)1003], and the Jiangsu Province Agricultural Science \& Technology Support Program (\#BE2013301).

\section{Supplementary Material}

\section{REFERENCES}

Bai X, Luo L, Yan W, Kovi MR, et al. (2010). Genetic dissection of rice grain shape using a recombinant inbred line population derived from two contrasting parents and fine mapping a pleiotropic quantitative trait locus qGL7. BMC Genet. 11: 16. 
Borevitz JO and Chory J (2004). Genomics tools for QTL analysis and gene discovery. Curr. Opin. Plant Biol. 7: 132-136.

Dixit N, Dokku P, Amitha Mithra SV, Parida SK, et al. (2013). Haplotype structure in grain weight gene GW2 and its association with grain characteristics in rice. Euphytica 192: 55-61.

Fan C, Xing Y, Mao H, Lu T, et al. (2006). GS3, a major QTL for grain length and weight and minor QTL for grain width and thickness in rice, encodes a putative transmembrane protein. Theor. Appl. Genet. 112: 1164-1171.

$\mathrm{Hu}$ Z, He H, Zhang S, Sun F, et al. (2012). A Kelch motif-containing serine/threonine protein phosphatase determines the large rain QTL trait in rice. J. Integr. Plant Biol. 54: 979-990.

Huang R, Jiang L, Zheng J, Wang T, et al. (2013). Genetic bases of rice grain shape: so many genes, so little known. Trends Plant Sci. 18: 218-226.

Ishimaru K, Hirotsu N, Madoka Y, Murakami N, et al. (2013). Loss of function of the IAA-glucose hydrolase gene TGW6 enhances rice grain weight and increases yield. Nat. Genet. 45: 707-711.

Li Y, Fan C, Xing Y, Jiang Y, et al. (2011). Natural variation in GS5 plays an important role in regulating grain size and yield in rice. Nat. Genet. 43: 1266-1269

Lu L, Shao D, Qiu X, Sun L, et al. (2013). Natural variation and artificial selection in four genes determine grain shape in rice. New Phytol. 200: 1269-1280.

Mao H, Sun S, Yao J, Wang C, et al. (2010). Linking differential domain functions of the GS3 protein to natural variation of grain size in rice. Proc. Natl. Acad. Sci. U.S.A. 107: 19579-19584.

Qi P, Lin YS, Song XJ, Shen JB, et al. (2012). The novel quantitative trait locus GL3.1 controls rice grain size and yield by regulating Cyclin-T1;3. Cell Res. 22: 1666-1680.

Shao G, Wei X, Chen M, Tang S, et al. (2012). Allelic variation for a candidate gene for GS7, responsible for grain shape in rice. Theor. Appl. Genet. 125: 1303-1312.

Shomura A, Izawa T, Ebana K, Ebitani T, et al. (2008). Deletion in a gene associated with grain size increased yields during rice domestication. Nat. Genet. 40: 1023-1028.

Song XJ, Huang W, Shi M, Zhu MZ, et al. (2007). A QTL for rice grain width and weight encodes a previously unknown RINGtype E3 ubiquitin ligase. Nat. Genet. 39: 623-630.

Takano-Kai N, Jiang H, Kubo T, Sweeney M, et al. (2009). Evolutionary history of GS3, a gene conferring grain length in rice. Genetics 182: 1323-1334.

Takano-Kai N, Doi K and Yoshimura A (2011). GS3 participates in stigma exsertion as well as seed length in rice. Breeding Sci. 61: 244-250.

Wang S, Wu K, Yuan Q, Liu X, et al. (2012). Control of grain size, shape and quality by OsSPL16 in rice. Nat. Genet. 44: 950954.

Weng J, Gu S, Wan X, Gao H, et al. (2008). Isolation and initial characterization of GW5, a major QTL associated with rice grain width and weight. Cell Res. 18: 1199-1209.

Xing Y and Zhang Q (2010). Genetic and molecular bases of rice yield. Annu. Rev. Plant Biol. 61: 421-442.

Yan S, Zou G, Li S, Wang H, et al. (2011). Seed size is determined by the combinations of the genes controlling different seed characteristics in rice. Theor. Appl. Genet. 123: 1173-1181.

Ying JZ, Gao JP, Shan JX, Zhu MZ, et al. (2012). Dissecting the genetic basis of extremely large grain shape in rice cultivar 'JZ1560'. J. Genet. Genomics 39: 325-333.

Zhang X, Wang J, Huang J, Lan H, et al. (2012). Rare allele of OsPPKL1 associated with grain length causes extra-large grain and a significant yield increase in rice. Proc. Natl. Acad. Sci. U.S.A. 109: 21534-21539.

Zhang YD, Zhang YH, Dong SL, Chen T, et al. (2013). QTL mapping for grain size traits based on extra-large grain rice line TD70. Rice Sci. 20: 400-406.

Zuo J and Li J (2014). Molecular genetic dissection of quantitative trait Loci regulating rice grain size. Annu. Rev. Genet. 48: 